\title{
Étude multilocale de blés hybrides : niveaux d'hétérosis et élaboration du rendement
}

\author{
FX Oury 1, P Brabant 2, P Pluchard 3, P Bérard 1, M Rousset 1 \\ Avec la collaboration technique de J Gourdon ${ }^{1}, \mathrm{H}_{\text {Corti }}{ }^{2}$, JP Meunier ${ }^{2}$, A Blake ${ }^{3}$ \\ 1 INRA, Station d'Amélioration des Plantes, Domaine de Crouelle, 63000 Clermont-Ferrand; \\ 2 INRA - UPS, Station de Génétique Végétale, Ferme du Moulon, 91190 Gif-sur-Yvette; \\ ${ }^{3}$ INRA, Domaine de Brunehaut, Estrées-en-Chaussées, 80200 Péronne, France
}

(Reçu le 8 mars 1989; accepté le 29 août 1990)

\begin{abstract}
Résumé - Une expérimentation multilocale nous a permis de comparer 35 hybrides de blé tendre d'hiver avec leurs parents, dans des conditions environnementales variées. Ce matériel a été évalué dans des dispositifs en blocs complets randomisés, la parcelle élémentaire étant au moins de $3,5 \mathrm{~m}^{2}$. Dans les 3 lieux, il y a un hétérosis pour le rendement, par rapport au meilleur parent, et les $F_{1}$ ont, en moyenne, des résultats supérieurs à ceux du parent moyen pour la hauteur et le poids de 1000 grains, proches de ceux du parent moyen pour le nombre d'épis par $\mathrm{m}^{2}$ et la fertilité de l'épi, et au niveau de ceux du parent le plus précoce. Les lignées parentales couvrant une gamme de valeurs agronomiques assez large, il apparait une relation positive entre le rendement de l'hybride et celui de ses parents, quel que soit le lieu. Dans nos conditions de culture intensive, l'homéostasie n'est pas plus importante chez les $F_{1}$ que chez les parents. Dans les 3 lieux, on constate que les capacités de remplissage de l'hybride sont plus importantes que celles de la lignée : à nombre de grains par $\mathrm{m}^{2}$ égal, l'hybride a un poids de 1000 grains plus élevé. Par contre, à nombre d'épis par $\mathrm{m}^{2}$ égal, une $F_{1}$ ne produit pas plus de grains par épi qu'une lignée, quel que soit le lieu. Pour le choix des parents d'hybrides, il paraît intéressant de privilégier des lignées de bonne valeur propre et qui produisent beaucoup de grains par $\mathrm{m}^{2}$.
\end{abstract}

blé hybride / hétérosis / homéostasie / composante du rendement

Summary - Multilocal analysis of wheat hybrids: levels of heterosis and yield elaboration. On a multilocal experiment, 35 winter wheat hybrids together with their parents were compared, under various environmental conditions. This material has been evaluated in randomised complete blocks designs, the elementary plot being $3.5 \mathrm{~m}^{2}$ minimum. In the 3 locations, high-parent heterosis is demonstrated for yield, and the $F_{1}$ are, on average, above the mid-parent for 1000 seed weight and height, close to the mid-parent for the number of ears per $\mathrm{m}^{2}$ and fertility of ear, and on a par with the earlier parent (table l, and figs 1-5). The hybrid yield is correlated with that of the parents in all locations (table II), which is probably due to a large scale of parental agronomical values. Under the intensive cultivation conditions of the experiment, homeostasis is not greater for $F_{1}$ than for inbred lines (tables III-VI). In the 3 locations, grain filling capacities of hybrids are greater than those of inbreds: with the same number of grains per $\mathrm{m}^{2}$, hybrids have a better 1000 seed weight (fig 7). On the other hand, with an identical number of ears per $m^{2}, F_{1}$ 's do not produce more grains per ear than inbreds (fig 6). For the selection of parental lines, it is interesting to select those with a good value per se, producing a large number of grains per $m^{2}$ (table VIII).

hybrid wheat / heterosis / homeostasis / yield factor

\section{INTRODUCTION}

Les possibilités d'expérimentation sur les blés hybrides se sont considérablement élargies depuis l'apparition des agents chimiques d'hybridation. Ces derniers permettent en effet de produire de nombreuses combinaisons à partir des meilleures variétés du moment (Borghi et al, 1989; Morgan et al, 1989), sans les inconvé- nients liés à l'utilisation de la stérilité mâle génocytoplasmique (Rousset, 1986; Glenn, 1987).

Cependant, le développement commercial des blés hybrides reste lié au potentiel de production des $F_{1}$ par rapport aux lignées, évalué dans une large gamme de conditions environnementales. En effet, dans l'état actuel des techniques de production, le coût de la semence hybride est plus élevé que celui de la semence de lignée 
pure. II faut donc que la productivité des $F_{1}$ soit supérieure à celle des lignées pour compenser ce surcoût.

Pour réaliser cet objectif dans un programme de sélection de blés hybrides, il nous a paru intéressant de comprendre comment se décompose le rendement d'un hybride par rapport à celui de ses parents, afin de dégager des critères de sélection en vue du développement d'hybrides très performants, stables et à fort hétérosis.

Dans un précédent article (Oury et al, 1990), nous avons présenté le bilan d'expérimentations faites à Clermont-Ferrand sur plusieurs années. Les hybrides étudiés étaient comparés à leurs lignées parentales, toutes de très bonne valeur agronomique.

Le présent article expose les résultats d'une expérimentation multilocale, mise en place dans le cadre de la collaboration entre I'INRA et le GIE "Hybriblé»". Les essais ont été conduits en 1989, sur les stations INRA de Clermont-Ferrand et Mons-en-Chaussée, ainsi qu'à la station de génétique végétale du Moulon. La variabilité des parents des hybrides testés, du point de vue de la valeur agronomique, était contrairement à l'étude précédente, assez étendue. En effet, notre but n'était pas de mettre en évidence les potentialités maximales des blés hybrides, mais d'obtenir une large gamme de valeurs propres afin de mieux appréhender le comportement des $F_{1}$. Nous avons étudié les manifestations de l'hétérosis pour différents caractères, et la décomposition du rendement chez les hybrides. L'analyse multilocale nous a permis de mettre en parallèle l'homéostasie observée chez les $F_{1}$, et celle observée chez les lignées parentales.

\section{MATÉRIEL ET MÉTHODES}

Nous avons mis en expérimentation 48 hybrides obtenus par voie chimique, et les 47 lignées parentales correspondantes. La plupart des hybrides ont été produits avec le gamétocide Orsan, à partir de lignées fournies par les différents membres du GIE "Hybriblé", et par les différents partenaires INRA de ce GIE. Pour compléter l'effectif, nous avons retenu en plus 9 hybrides provenant de croisements entre des lignées INRA (gamétocide Nickerson), et 2 hybrides inscrits au catalogue (gamétocide Rhom et Haas). L'origine diversifiée du matériel végétal, a conduit à l'obtention d'un échantillon de lignées parentales qui couvrait une gamme de valeurs propres assez étendue.
Le taux de pureté des $F_{1}$ a été déterminé par électrophorèse des gluténines, et nous n'avons retenu pour notre étude que les combinaisons pour lesquelles le taux de plantes de type femelle ou hors-type était inférieur à $30 \%$. Ce seuil a été déterminé lors d'une étude antérieure ( $P$ Brabant, non publié), comme étant celui en dessous duquel le jugement des performances des hybrides n'était pas (ou très peu) influencé par leur degré d'impureté. En définitive, nous avons conservé 35 hybrides (dont le taux moyen de pureté était de $87 \%$, avec des valeurs extrêmes de $71 \%$ et $100 \%$ ), et leurs 37 parents (certaines lignées parentales ont été utilisées plus d'une fois pour la fabrication des $F_{1}$ : parmi les 27 femelles, 2 intervenaient dans 3 combinaisons hybrides, et 6 autres dans 2 combinaisons; parmi les 10 mâles, 5 intervenaient dans respectivement $8,7,5,4$ et 3 combinaisons hybrides, et 3 autres dans 2 combinaisons).

Un des parents était absent de l'expérimentation de Clermont, et 4 parents manquaient dans l'expérimentation du Moulon. Pour ces 2 lieux, les calculs d'hétérosis n'ont donc pu être réalisés pour les $F_{1}$ dans lesquelles ces lignées intervenaient ( 1 hybride à Clermont; 7 hybrides au Moulon).

Le dispositif expérimental commun aux 3 lieux comprenait 3 blocs complets randomisés. La parcelle élémentaire était constituée de 6 lignes de $5 \mathrm{~m}$ espacées de $20 \mathrm{~cm}$ à Clermont et à Mons, et de 6 lignes de 2,1 m espacées de $23 \mathrm{~cm}$ au Moulon. Les techniques culturales appliquées en chacun des lieux correspondaient aux conduites intensives couramment mises en œuvre dans ces différentes régions (en particulier des apports en N, P, et K, ajustés à des objectifs de rendement élevés; une couverture fongicide et insecticide efficace). Les expérimentations de Clermont et Mons ont, de plus, reçu un traitement raccourcisseur.

Les variables mesurées ont été le rendement en grains à $15 \%$ d'humidité (RDT), le nombre d'épis par $\mathrm{m}^{2}$ (NE), le poids de 1000 grains à $15 \%$ d'humidité (PMG), la hauteur $(H T)$ et la précocité d'épiaison (PREC). Le nombre de grains par $\mathrm{m}^{2}$ (NG) a ensuite été calculé à partir de $R D T$ et $P M G$, ainsi que le nombre de grains par épi (NGE), à partir de NG et NE. L'ensemble de ces données était disponible pour Clermont et le Moulon, mais les données relatives à $N E$ (et donc NGE), HT et PRÉC, manquaient pour Mons, où ces 3 caractères n'ont pas été mesurés.

Pour étudier l'interaction "génotype $x$ milieu» nous avons utilisé le modèle d'analyse de variance suivant, dans lequel tous les facteurs sont considérés comme fixés :

$y_{i j k}=m+l_{i}+b_{i j}+g_{k}+l_{i} \cdot g_{k}+E_{i j k}$

où $m=$ moyenne générale

$l_{i}=$ effet «lieu"

$b_{i j}=$ effet "bloc" hiérarchisé à l'effet ulieu"

$g_{k}=$ effet «génotype»

$l_{i} \cdot g_{k}=$ interaction "génotype $x$ lieu"

$E_{i j k}=$ erreur résiduelle

* Le GIE "Hybrible" regroupe les établissements de sélection Blondeau, Momont, Orsem et UCASP, et la firme Orsan (pour la recherche sur les gamétocides). 
Pour comparer 2 régressions effectuées, l'une avec les données concernant les hybrides, l'autre avec celles concernant les parents, nous avons utilisé la méthode décrite par Tomassone et al (1983). Elle nécessite de réaliser sur l'ensemble des données (hybrides + parents), d'une part une régression simple, et d'autre part une régression multiple intégrant 2 variables indicatrices qui permettent de distinguer les 2 sous-populations. Un test de Fisher, calculé à partir des valeurs des ccefficients de détermination simple et multiple, permet alors de savoir si les régressions relatives aux hybrides et aux lignées sont significativement différentes ou pas.

Les traitements statistiques ont été réalisés à l'aide des logiciels «Amance" (Bachacou et al, 1981), et "Consistent System" (avec son gestionnaire de bases de données “Janus", et son interface graphique "SIGS").

\section{RÉSULTATS}

Le niveau des rendements a été élevé en chacun des lieux : les moyennes réalisées ont été de $82,7 \mathrm{q} / \mathrm{ha}$ à Clermont, 94,3 $\mathrm{q} / \mathrm{ha}$ au Moulon et $89,3 \mathrm{q} / \mathrm{ha}$ à Mons. La précision des essais pour le rendement était bonne : les cœefficients de variation on été de $4,3 \%$ à Clermont, de $5,8 \%$ au Moulon et de $3,9 \%$ à Mons.

Le rendement moyen des hybrides a été de $86,1 \mathrm{q} / \mathrm{ha}$ à Clermont, 98,7 $\mathrm{q} / \mathrm{ha}$ au Moulon et 93,3 q/ha à Mons, pour un rendement moyen des parents respectivement de $79,5 \mathrm{q} / \mathrm{ha}, 91,3 \mathrm{q} / \mathrm{ha}$ et $85,8 \mathrm{q} / \mathrm{ha}$, et un rendement moyen des témoins (Camp-Rémy, Festival, Promentin et Thésée) respectivement de $87,2,98,3$ et $94 \mathrm{q} / \mathrm{ha}$.

\section{Héterosis pour les différents caractères étudiés}

Nous avons vérifié qu'il n'existait pas de corrélation entre l'hétérosis et le taux de pureté, pour l'échantillon d'hybrides que nous avons étudié.

Dans les 3 lieux, la plupart des hybrides sont supérieurs au parent moyen pour RDT, PMG et $H T$ (tableau I, figs 1 et 2). C'est pour ces 3 variables que l'hétérosis par rapport au parent moyen est le plus important (un contraste "parents-hybrides" à 1 ddl, en analyse de variance, montre que, dans les 3 lieux, la supériorité des $F_{1}$ par rapport aux lignées pour $R D T$, $P M G$ et $H T$, est hautement significative, $P<$ $0,01)$. Cependant, un hétérosis par rapport au parent supérieur n'apparaît que dans le cas de $R D T$. Pour $N E, N G$ et $N G E$, les $F_{1}$ sont proches du parent moyen (tableau I, figs 3 et 4), avec tou- tefois un hétérosis par rapport au parent moyen pour NG et NGE plus élevé au Moulon et à Mons qu'à Clermont (le contraste "parentshybrides" en analyse de variance, indique qu'il n'y a aucune différence significative entre $F_{1}$ et lignées pour NE, NG et NGE, sauf au Moulon où les hybrides sont significativement supérieurs aux parents pour NGE, $P<0,01$ ). Par ailleurs, la plupart des hybrides sont plus précoces que le parent moyen (tableau l et fig 5).

Tous ces résultats concernant les niveaux d'hétérosis pour les différentes variables, vont dans le même sens que ceux déjà obtenus dans des expérimentations faisant intervenir un nombre relativement important d'hybrides observés en parcelles de plusieurs $\mathrm{m}^{2}$, que ces hybrides aient été produits manuellement (Goujon et Paquet, 1968; Keydel, 1973), ou par voie chimique (Borghi et al, 1988; Morgan et al, 1989; Oury et al, 1990).

Les figures 1,3 et 4, montrent que pour les composantes du rendement NG, NGE et PMG, la valeur maximale prise par les $F_{1}$ est du même ordre de grandeur que celle prise par les parents. Par contre, pour NE, ce sont des lignées qui obtiennent les valeurs les plus fortes, et il n'y a pas d'hybrides dans la zone des forts nombres d'épis par $\mathrm{m}^{2}$. En revanche, pour $R D T$, quelques $F_{1}$ dépassent nettement les meilleures lignées : le meilleur hybride a un rendement supérieur à celui de la meilleure lignée, de 6,9\% à Clermont, de $6,3 \%$ au Moulon et de 6,4\% à Mons.

Les figures 2 et 5 montrent que les $F_{1}$ sont proches du parent le plus précoce, et proches du parent le plus haut. Les données de Clermont pour la taille des plantes sont cependant à considérer avec précaution, compte tenu d'éventuelles interactions "génotype $x$ raccourcisseur". En conséquence, pour notre échantillon de génotypes, les gammes de tailles et de précocités décrites par les hybrides sont sensiblement les mêmes que celles décrites par les lignées parentales.

\section{Lien entre valeur des hybrides. et valeur des parents}

Pour les 3 lieux, on constate qu'il y a une relation positive entre le rendement de l'hybride d'une part, et le rendement du parent moyen ou du parent supérieur d'autre part (tableau II).

Clermont se distingue des 2 autre lieux par le fait que le rendement de l'hybride est fortement corrélé à l'hétérosis parent supérieur pour le ren- 
Tableau I. Hétérosis par rapport aux parents moyen et supérieur, pour les 7 caractères étudiés $(R D T=$ rendement en grains; $N E=$ nombre d'épis par $\mathrm{m}^{2} ; N G=$ nombre de grains par $\mathrm{m}^{2} ; N G E=$ nombre de grains par épi; $P M G=$ poids de 1000 grains; $H T=$ hauteur; $P R E E C=$ précocité $)$, et les 3 lieux d'expérimentation $(a=$ Clermont; $b=$ Le Moulon; $c=$ Mons).

\begin{tabular}{|c|c|c|c|c|c|c|c|}
\hline & & \multicolumn{3}{|c|}{$\begin{array}{c}\text { Hétérosis } \\
\text { (\% parent moyen) }\end{array}$} & \multicolumn{3}{|c|}{$\begin{array}{c}\text { Hétérosis } \\
\text { (\% parent supérieur) }\end{array}$} \\
\hline & & $\min i$ & moyen & $\max i$ & $\operatorname{mini}$ & moyen & $\max i$ \\
\hline$R D T$ & $\begin{array}{l}a \\
b \\
c\end{array}$ & $\begin{array}{l}-2 \\
-0,8 \\
-1,4\end{array}$ & $\begin{array}{l}9 \\
9,6 \\
9,3\end{array}$ & $\begin{array}{l}20,6 \\
23,5 \\
20\end{array}$ & $\begin{array}{l}-5,4 \\
-8,6 \\
-7,4\end{array}$ & $\begin{array}{l}4 \\
4,5 \\
4,7\end{array}$ & $\begin{array}{l}15,9 \\
19,3 \\
17,4\end{array}$ \\
\hline$N E$ & $\begin{array}{l}a \\
b \\
c\end{array}$ & $\begin{array}{c}-11,4 \\
-14,8 \\
-\end{array}$ & $\begin{array}{r}1,6 \\
-1,2 \\
-\end{array}$ & $\begin{array}{c}23,2 \\
11,7 \\
-\end{array}$ & $\begin{array}{c}-22,4 \\
-19,7 \\
-\end{array}$ & $\begin{array}{l}-4,8 \\
-5,3 \\
-\end{array}$ & $\begin{array}{c}21,1 \\
10,7 \\
-\end{array}$ \\
\hline$N G$ & $\begin{array}{l}a \\
b \\
c\end{array}$ & $\begin{array}{r}-6,6 \\
7,4 \\
-9,2\end{array}$ & $\begin{array}{l}1,3 \\
3,9 \\
3,4\end{array}$ & $\begin{array}{l}12,3 \\
13,2 \\
22,5\end{array}$ & $\begin{array}{l}-17,9 \\
-20,1 \\
-15,5\end{array}$ & $\begin{array}{l}-6,6 \\
-3,5 \\
-3,1\end{array}$ & $\begin{array}{r}11,2 \\
9,1 \\
14,5\end{array}$ \\
\hline NGE & $\begin{array}{l}a \\
b \\
c\end{array}$ & $\begin{array}{c}-19,2 \\
-13,7 \\
-\end{array}$ & $\begin{array}{c}-0,7 \\
5,3 \\
-\end{array}$ & $\begin{array}{c}14,3 \\
17,1 \\
-\end{array}$ & $\begin{array}{c}-15,4 \\
-27,8 \\
-\end{array}$ & $\begin{array}{c}-11,1 \\
-1,6 \\
-\end{array}$ & $\begin{array}{c}10,3 \\
16,8 \\
-\end{array}$ \\
\hline$P M G$ & $\begin{array}{l}a \\
b \\
c\end{array}$ & $\begin{array}{l}-4,3 \\
-1,3 \\
-4,2\end{array}$ & $\begin{array}{l}6,9 \\
5 \\
5,3\end{array}$ & $\begin{array}{l}18,9 \\
15 \\
16,6\end{array}$ & $\begin{array}{l}-15,1 \\
-15,9 \\
-13,7\end{array}$ & $\begin{array}{r}0,7 \\
-0,7 \\
-1\end{array}$ & $\begin{array}{l}18,6 \\
13,1 \\
12,6\end{array}$ \\
\hline$H T$ & $\begin{array}{l}a \\
b \\
c\end{array}$ & $\begin{array}{r}-5,9 \\
1,7 \\
-\end{array}$ & $\begin{array}{l}4,6 \\
7,7 \\
-\end{array}$ & $\begin{array}{c}17,2 \\
19,3 \\
-\end{array}$ & $\begin{array}{c}-11,1 \\
-9,8 \\
-\end{array}$ & $\begin{array}{r}-1,4 \\
1,7 \\
-\end{array}$ & $\begin{array}{c}13,3 \\
13,8 \\
-\end{array}$ \\
\hline PRÉC & $\begin{array}{l}a \\
b \\
c\end{array}$ & $\begin{array}{c}-2,8 \\
-1,7 \\
-\end{array}$ & $\begin{array}{c}-0,7 \\
-0,6 \\
-\end{array}$ & $\begin{array}{l}0,7 \\
0,2 \\
-\end{array}$ & $\begin{array}{l}-5,7 \\
-3,7 \\
-\end{array}$ & $\begin{array}{l}-1,8 \\
-1,4 \\
-\end{array}$ & $\begin{array}{l}0 \\
0,2 \\
-\end{array}$ \\
\hline
\end{tabular}

dement, cet hétérosis étant, par ailleurs, indépendant du niveau de rendement du parent supérieur. Au Moulon et à Mons, au contraire, l'hétérosis parent supérieur pour le rendement est corrélé négativement au rendement du parent supérieur, et la liaison entre le rendement de l'hybride et cet hétérosis est très faible.

\section{Comparaison des stabilités inter-lieux des hybrides et des parents}

II y a un fort effet «lieu», et une interaction "génotype x lieu" significative, pour RDT, NG et $P M G$, que le modèle d'analyse de variance soit appliqué aux $F_{1}$ ou aux parents (tableau III). De plus, les carrés moyens de cette interaction sont du même ordre de grandeur pour les hybrides et pour les lignées. Si on considère les classements obtenus par les hybrides dans les différents lieux, on s'aperçoit d'une part qu'ils ne sont pas plus corrélés entre eux que ne le sont les classements obtenus par les parents dans ces mêmes lieux, et d'autre part, que les corrélations varient de la même façon pour les hybrides et pour les lignées, lorsqu'on considère les lieux 2 à 2 (tableau IV). Par ailleurs, les moyennes des estimations des écarts-types interlieux pour les différentes variables étudiées, sont tout à fait comparables pour les $F_{1}$ et pour les lignées (tableau V).

Tous ces résultats indiquent que, dans les conditions de notre expérimentation, les hybrides et les lignées réagissent de la même manière 

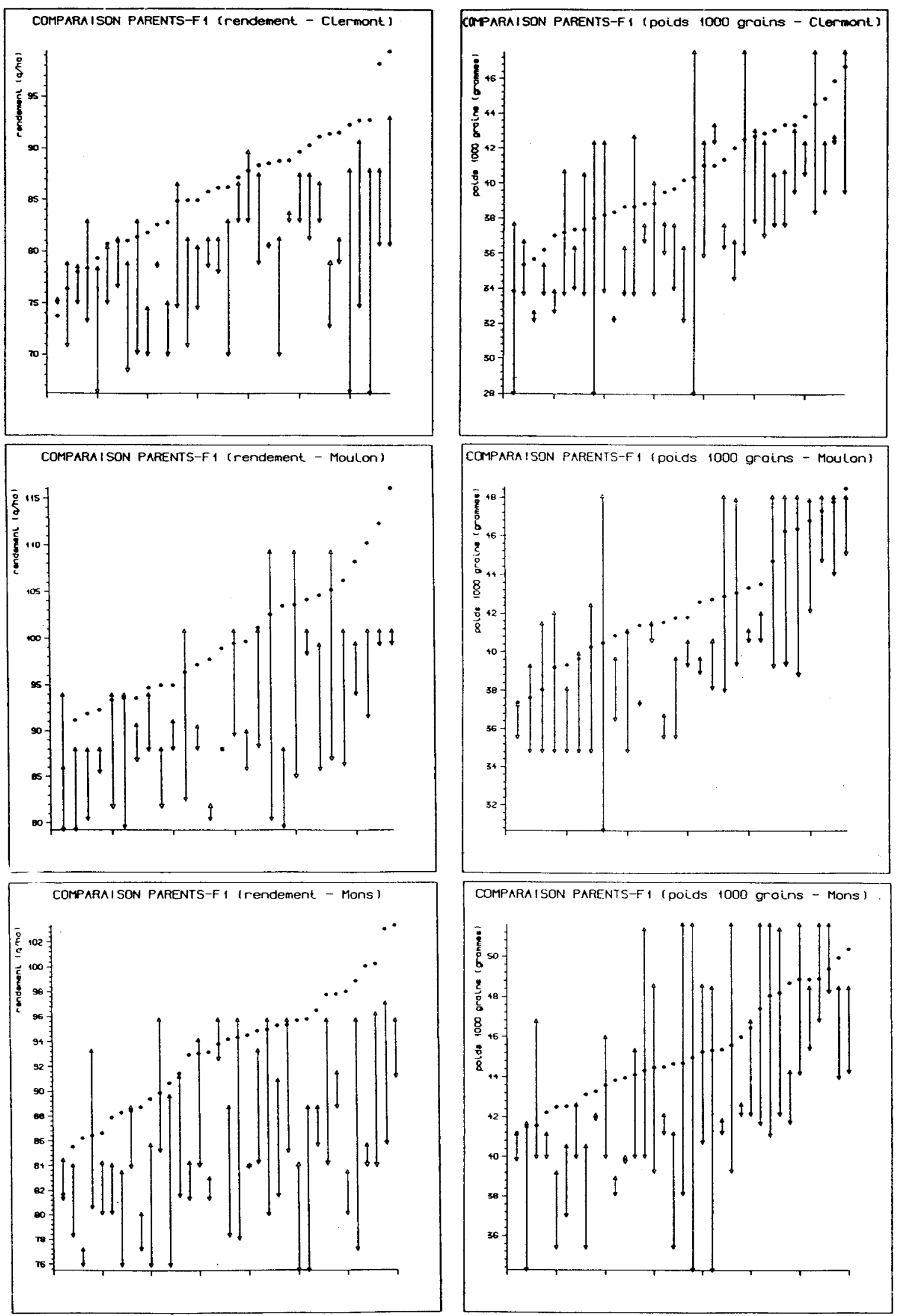

Fig 1. Valeurs observées pour les hybrides ( $\left.{ }^{*}\right)$, leurs parents inférieurs $(\nabla)$ et leurs parents supérieurs $(\Delta)$, pour 2 variables (RDT et $P M G$ ) pour lesquelles se manifeste un hétérosis par rapport au parent moyen dans les 3 lieux. Les triplets ahybride + parents" sont disposés selon les valeurs croissantes des $F_{1}$. 


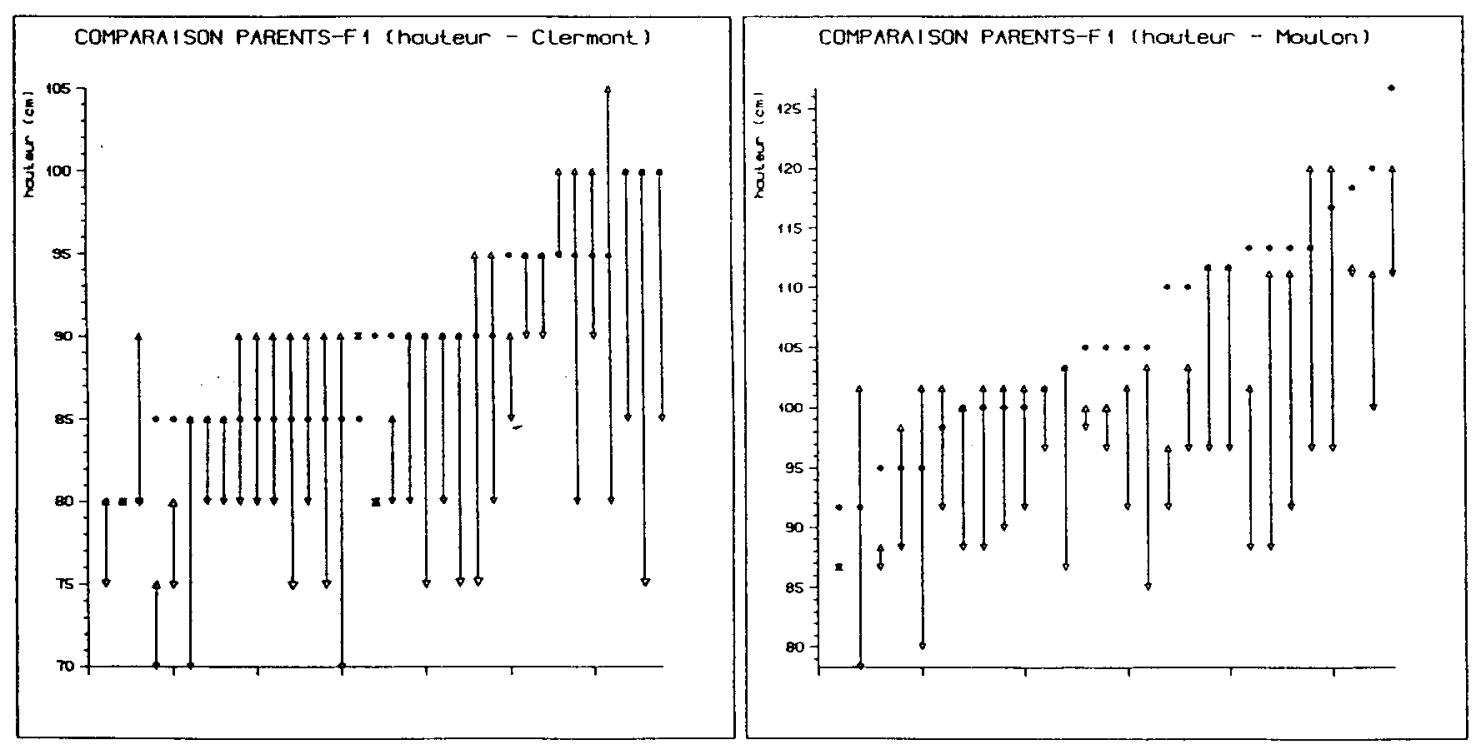

Fig 2. Hauteur des hybrides ( $\left.{ }^{*}\right)$, de leurs parents inférieurs $(\nabla)$ et de leurs parents supérieurs $(\Delta)$, en 2 lieux (Clermont et le Moulon). Les triplets «hybride + parents" sont disposés selon les valeurs croissantes des $F_{1}$.

aux variations environnementales, et ont donc des stabilités interlieux équivalentes.

Lorsqu'on s'intéresse aux hétérosis pour $R D T$, $N G$ et $P M G$, on constate qu'il y a également une interaction "génotype $x$ lieu" significative, qu'il s'agisse de l'hétérosis par rapport au parent moyen ou de l'hétérosis par rapport au parent supérieur (tableau VI). Par contre, l'effet "lieu» disparaît, ce qui correspond au fait que les niveaux moyens d'hétérosis sont du même ordre de grandeur dans les différents lieux. Par ailleurs, les écarts types interlieux des valeurs d'hétérosis sont, en moyenne, assez élevés (tableau V). Les niveaux d'hétérosis, pour une combinaison hybride donnée, sont donc généralement variables d'un lieu à l'autre pour tous les caractères étudiés, excepté la précocité.

La stabilité interlieux n'apparaît pas liée à la valeur des hybrides (tableau VII). Les corrélations les plus fortes relevées dans ce tableau indiquent même que les $F_{1}$ qui ont les rendements les plus élevés au Moulon, ont tendance à être les plus instables entre lieux.

\section{Modes d'élaboration du rendement des hybrides et des parents}

La régression de NGE sur NE montre que la relation de compensation entre ces 2 composantes existe aussi bien chez les lignées que chez les $F_{1}$. De plus, c'est une relation assez forte : les ccefficients de détermination sont de 0,579 et 0,307 à Clermont, et de 0,391 et 0,534 au Moulon, respectivement pour les parents et pour les hybrides. Par ailleurs, l'équation de la droite qui caractérise les hybrides n'est pas significativement différente de celle qui caractérise les lignées $\left(F_{2,67}=0,398\right.$ à Clermont: $P>0,05$; $F_{2,64}=0,656$ au Moulon : $P>0,05$ ). Pour un même nombre d'épis par $\mathrm{m}^{2}$, un hybride ne produit donc pas plus de grains par $\mathrm{m}^{2}$ qu'une lignée (fig 6).

Par contre, la régression de PMG sur NG permet de distinguer la population des hybrides de celle des parents, et cela pour les 3 lieux (fig 7). Le modèle de régression multiple basé sur la distinction de ces 2 populations, permet en effet une meilleure analyse de la relation entre les 2 variables que le modèle de régression linéaire simple appliqué à l'ensemble des génotypes $\left(F_{2,67}=10,63\right.$ à Clermont: $P<0,01 ; F_{2,64}=8,03$ au Moulon : $P<0,01 ; F_{2,68}=16,68$ à Mons : $P<$ $0,01)$. Pour un même nombre de grains par $\mathrm{m}^{2}$, un hybride remplit donc mieux ses grains qu'une lignée. Par ailleurs, le parallèlisme entre les 2 droites de régression montre que la compensation entre $N G$ et $P M G$ est similaire pour les $F_{1}$ et pour les parents, quel que soit le lieu. De plus, la relation entre ces 2 composantes est forte, car les cœfficients de détermination sont de 0,475 et 0,624 à Clermont, 0,416 et 0,365 au Moulon, et 0,601 et 0,468 à Mons, respectivement pour les parents et pour les hybrides. 

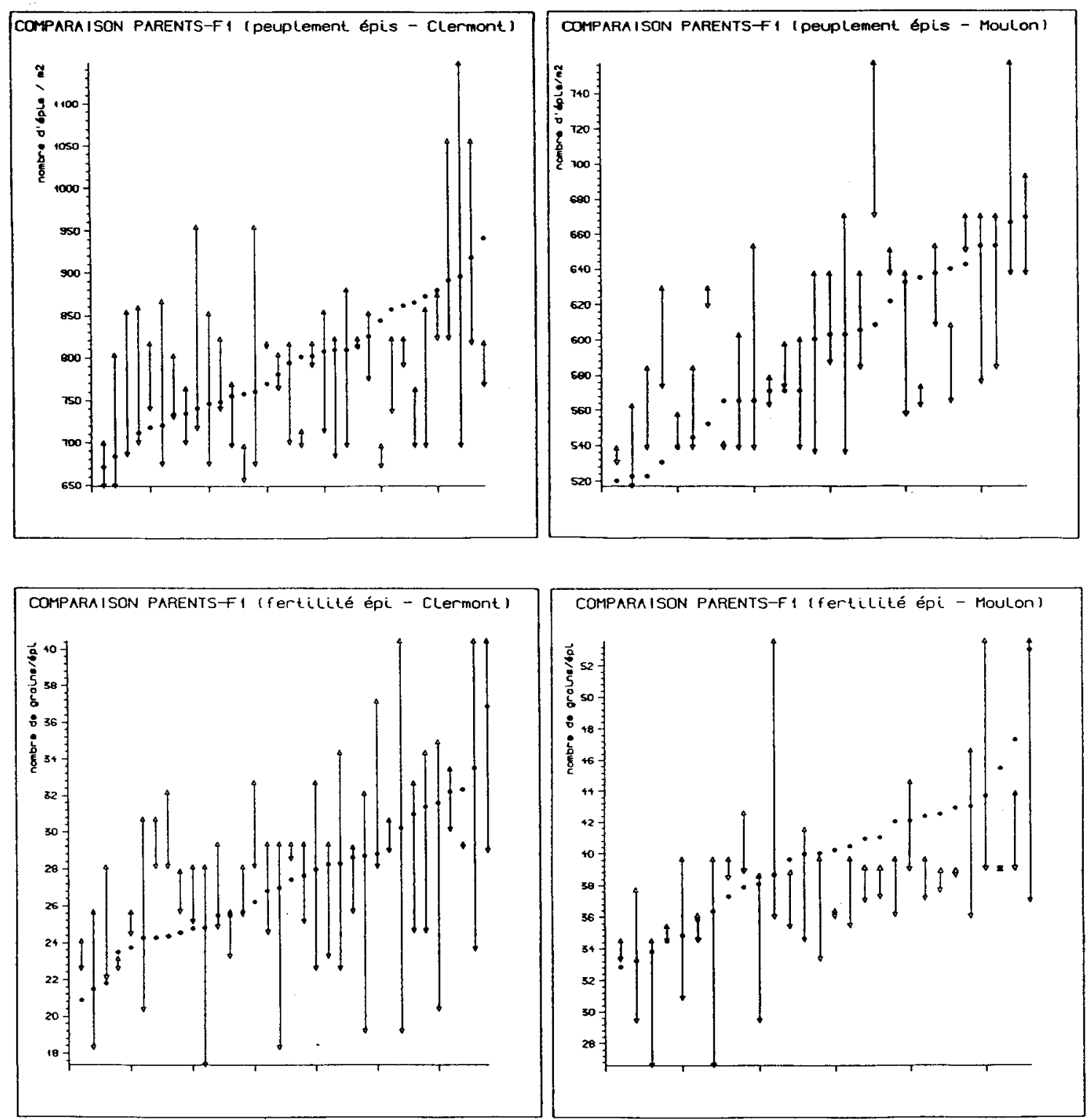

Fig 3. Nombre d'épis par m2 et fertilité de l'épi, des hybrides ("), de leurs parents inférieurs $(\nabla)$ et de leurs parents supérieurs $(\Delta)$, en 2 lieux (Clermont et le Moulon).

Les triplets «hybride + parents» sont disposés selon les valeurs croissantes des $F_{1}$.

Une étude des corrélations entre le rendement et ses différentes composantes montre que, dans les 3 lieux, il n'y a pas de relation entre RDT et $N E$, ou entre RDT et PMG, et cela aussi bien pour les hybrides que pour les lignées (tableau VIII). Par contre, il y a une corrélation positive entre RDT et NG, ainsi qu'entre RDT et NGE, et il y a une tendance au renforcement de ces relations lorsqu'on passe des parents aux $F_{1}$.

Par ailleurs, l'analyse des corrélations entre l'hétérosis pour le rendement et l'hétérosis pour les différentes composantes, montre que, sur l'ensemble des lieux, c'est la relation entre hété- rosis $R D T$ et hétérosis $N G$ qui est la plus forte et la plus stable (tableau VIII).

\section{DISCUSSION ET CONCLUSION}

\section{Prédiction de la valeur de l'hybride}

La relation que nous avons constatée entre le rendement de l'hybride et le rendement de ses parents paraît suffisamment forte et suffisamment stable entre lieux pour être utilisable. Mor- 

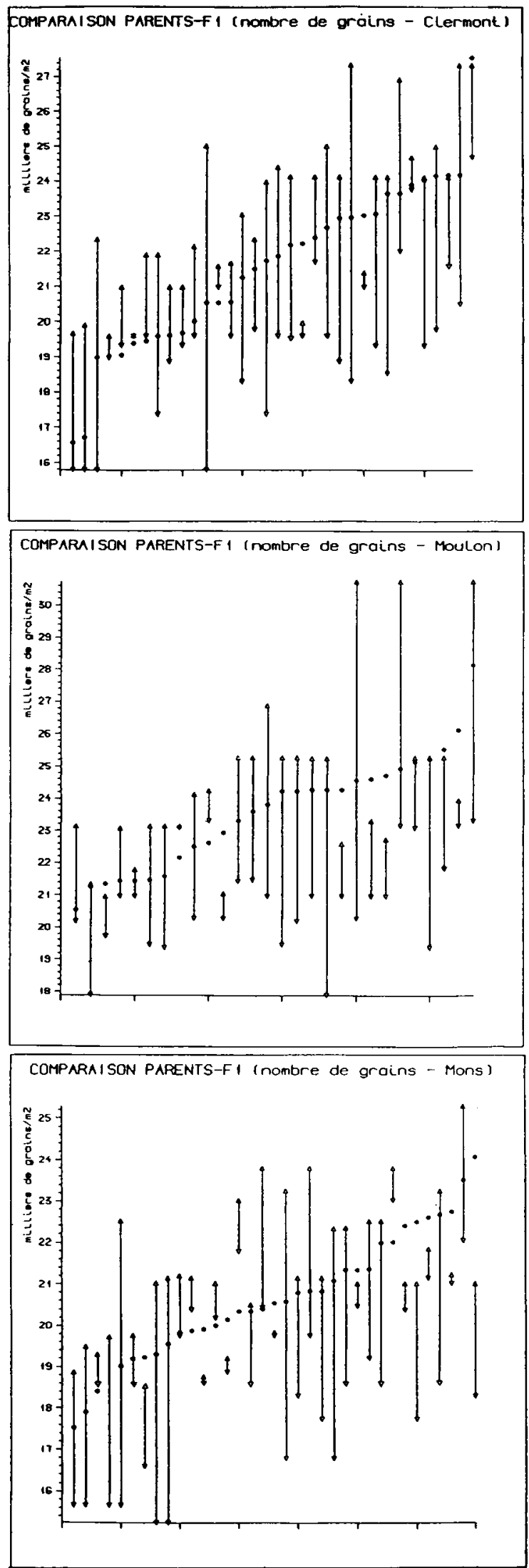

Fig 4. Nombre de grains par $\mathrm{m}^{2}$ produits par les hybrides $\left({ }^{*}\right)$, leurs parents inférieurs $(\nabla)$ et leurs parents supérieurs $(\Delta)$, dans les 3 lieux.

Les triplets whybride + parents" sont disposés selon les valeurs croissantes des $F_{1}$ gan et al (1989), en travaillant sur un large échantillon (430 hybrides étudiés), ont d'ailleurs mis en évidence une relation du même type. Notre étude confirme donc que pour obtenir des $F_{1}$ de bonne valeur propre, il est nécessaire de partir de parents qui ont eux-mêmes une bonne valeur propre. Cependant, cette relation ne permet qu'un tri assez grossier des candidats à l'hybridation, et il reste difficile de prédire le rendement de la $F_{1}$ à partir de celui de ses parents lorsque l'échantillon parental est constitué de lignées dont le niveau de performance est assez élevé et assez homogène (Oury et al, 1990).

En ce qui concerne l'étude de l'élaboration du rendement, nos résultats obtenus en expérimentation multilocale confirment ceux de Oury et al (1990) obtenus en un seul lieu (Clermont). On retrouve les mêmes corrélations négatives entre $N G$ et $P M G$, et entre NE et NGE, tant chez les hybrides que chez les lignées. Les relations de compensation entre composantes qui interviennent au cours de l'élaboration du rendement, lorsqu'il y a compétition entre plantes (Triboi et al, 1985; Triboi, 1986), ne sont donc pas différentes en situation hybride. On retrouve également, dans les 3 lieux, le phénomène de meilleur remplissage du grain commun à l'ensemble des $F_{1}$. Les capacités de remplissage des hybrides, à nombres de grains par $\mathrm{m}^{2}$ équivalents, sont donc plus importantes que celles des lignées.

La relation entre RDT et $N G$ (et aussi, dans la mesure où il existe une relation entre le rendement et l'hétérosis pour le rendement, la relation entre l'hétérosis pour RDT et l'hétérosis pour NG), semble suffisamment forte et stable entre lieux pour être exploitée. Cela peut se faire au travers de l'information éventuellement disponible au niveau des parents pour la composante «nombre de grains par $\mathrm{m}^{2}$ ". En effet, le nombre de grains produits par l'hybride est fortement corrélé au nombre de grains produits par le parent moyen (le cœefficient de corrélation est de 0,897 à Clermont, de 0,677 au Moulon et de 0,660 à Mons), ainsi qu'au nombre de grains produits par le parent supérieur (le coefficient de corrélation vaut 0,794 à Clermont, 0,647 à Moulon et 0,561 à Mons).

En définitive, et bien que cette approche reste assez grossière, on peut retenir qu'il paraît intéressant, au niveau du choix des candidats à l'hybridation, de privilégier des parents ayant une bonne valeur propre et produisant beaucoup de grains par $\mathrm{m}^{2}$. 


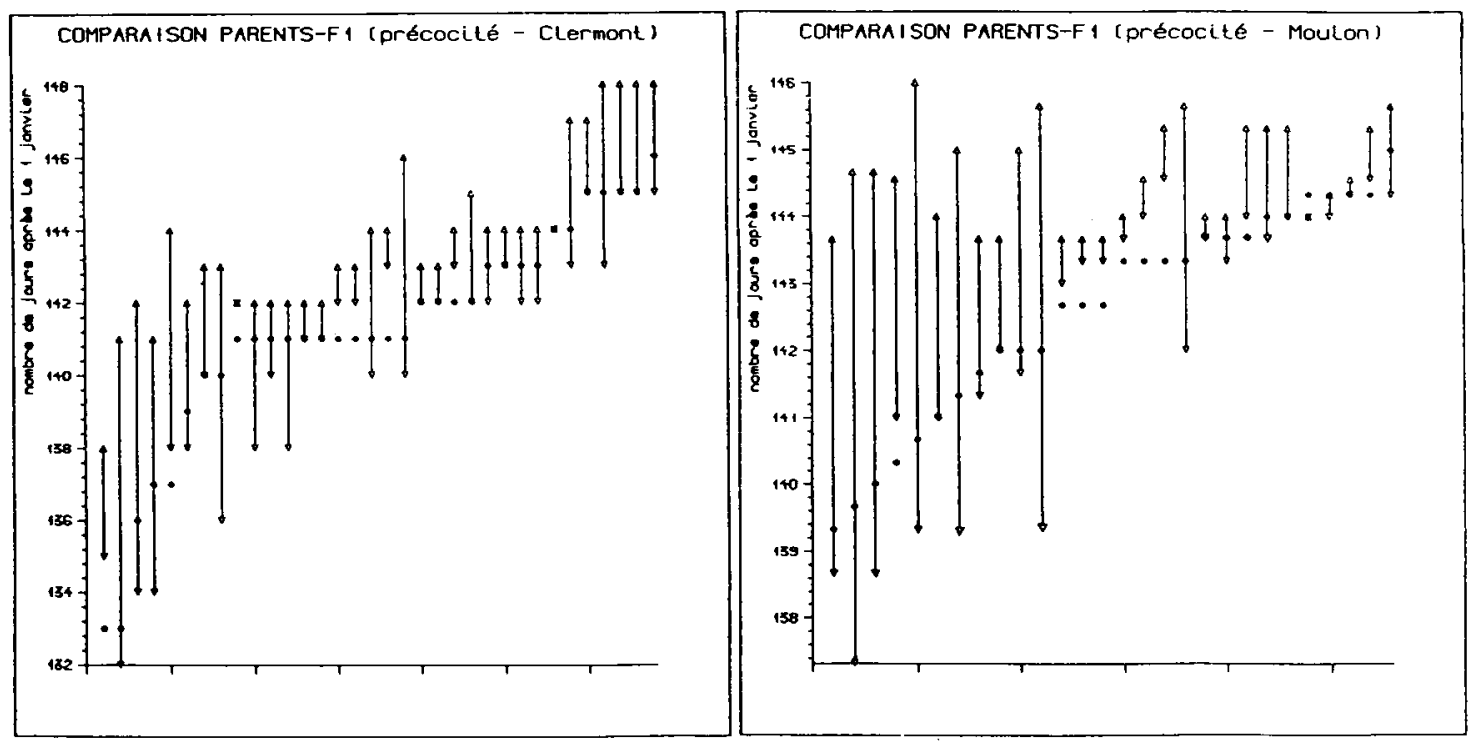

Fig 5. Précocité d'épiaison pour les hybrides (*), leurs parents inférieurs $(\nabla)$ et leurs parents supérieurs $(\Delta)$, en 2 lieux (Clermont et Le Moulon).

Les triplets «hybride + parents sont disposés selon les valeurs croissantes des $F_{1}$

Tableau II. Caractéristiques de différentes régressions linéaires mettant en évidence les liens existant entre le rendement de l'hybride, l'hétérosis pour le rendement et le rendement des parents.

NS = non significatif; ${ }^{*}=$ significatif au seuil de $5 \% ;{ }^{* \star}=$ significatif au seuil de $1 \% ;{ }^{\star \star \star}=$ significatif au seuil de $1 \%$; a = Clermont; $b=$ Le Moulon; $c=$ Mons.

\begin{tabular}{|c|c|c|c|}
\hline $\begin{array}{l}\text { Variable } \\
\text { expliquée } \\
(y)\end{array}$ & $\begin{array}{l}\text { Variable } \\
\text { explicative } \\
\quad(x)\end{array}$ & $\begin{array}{c}\text { Équation de la } \\
\text { droite de régression } \\
\text { (niveau de signification) }\end{array}$ & $\begin{array}{c}\text { Coff de } \\
\text { détermination } \\
\text { (coeff de corrélation) }\end{array}$ \\
\hline $\begin{array}{l}\text { rendement de } \\
\text { l'hybride }\end{array}$ & $\begin{array}{l}\text { hétérosis } \\
\text { parent supérieur }\end{array}$ & $\begin{array}{c}y=13,9+0,695 x \\
\left(^{* * *}\right) \\
y=59,1+0,389 x \\
\left(^{*}\right) \\
y=59,9+0,318 x \\
\left(^{*}\right)\end{array}$ & $\begin{array}{c}0,339 \\
(0,582) \\
0,153 \\
(0,391) \\
0,143 \\
(0,378)\end{array}$ \\
\hline $\begin{array}{l}\text { hétérosis } \\
\text { parent supérieur }\end{array}$ & $\begin{array}{l}\text { rendement } \\
\text { parent supérieur }\end{array}$ & 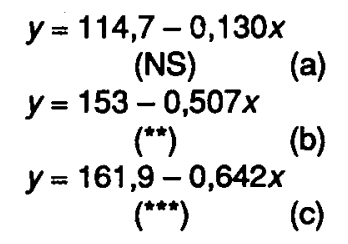 & $\begin{array}{c}0,015 \\
(-0,122) \\
0,275 \\
(-0,524) \\
0,345 \\
(-0,587)\end{array}$ \\
\hline $\begin{array}{l}\text { rendement de } \\
\text { l'hybride }\end{array}$ & $\begin{array}{l}\text { rendement } \\
\text { parent moyen }\end{array}$ & $\begin{array}{c}y=16,1+0,885 x \\
\left({ }^{* * *}\right) \\
y=10,1+0,984 x \\
(* *) \\
y=37,4+0,654 x \\
(* *)\end{array}$ & $\begin{array}{c}0,396 \\
(0,629) \\
0,587 \\
(0,766) \\
0,308 \\
(0,555)\end{array}$ \\
\hline $\begin{array}{l}\text { rendement de } \\
\text { l'hybride }\end{array}$ & $\begin{array}{l}\text { rendement } \\
\text { parent supérieur }\end{array}$ & $\begin{array}{c}y=8,4+0,938 x \\
(* *) \\
y=46,6+0,555 x \\
\left(^{* \star}\right) \\
y=50,1+0,483 x \\
\left(^{* *}\right)\end{array}$ & $\begin{array}{c}0,541 \\
(0,735) \\
0,332 \\
(0,576) \\
0,276 \\
(0,525)\end{array}$ \\
\hline
\end{tabular}


Tableau IIl. Analyses de variance sur les données parentales des 3 lieux; entre parenthèses, même chose pour les données relatives aux hybrides. ${ }^{*}=$ significatif au seuil de $5 \% ;{ }^{\star \star}=$ significatif au seuil de $1 \%$

\begin{tabular}{|c|c|c|c|c|c|}
\hline & $\begin{array}{c}\text { lieu } \\
2 d d l \\
(2 d d)\end{array}$ & $\begin{array}{c}\text { bloc } \\
6 d d l \\
(6 d d l)\end{array}$ & $\begin{array}{l}\text { Carrés moyens } \\
\text { génotype } \\
32 \text { ddl } \\
\text { (34 ddl) }\end{array}$ & $\begin{array}{c}\text { interaction } \\
\text { lieu-génotype } \\
59 \text { ddl } \\
(68 d d)\end{array}$ & $\begin{array}{c}\text { erreur } \\
182 d d l \\
(203 d d l)\end{array}$ \\
\hline$R D T$ & $\begin{array}{c}2291^{\star *} \\
\left(4155^{* \star}\right)\end{array}$ & $\begin{array}{l}146^{* *} \\
\left(251^{* *}\right)\end{array}$ & $\begin{array}{l}237^{* *} \\
\left(194^{* *}\right)\end{array}$ & $\begin{array}{l}86^{* *} \\
\left(63^{* \star}\right)\end{array}$ & $\begin{array}{c}18 \\
(19)\end{array}$ \\
\hline$N G$ & $\begin{array}{c}127441460^{* *} \\
\left(175209570^{* *}\right)\end{array}$ & $\begin{array}{l}7080111^{* *} \\
\left(9996807^{* *}\right)\end{array}$ & $\begin{array}{c}40333186 \text { "**} \\
(25897608 \text { **) }\end{array}$ & $\begin{array}{c}5232843^{* \star} \\
\left(4223084^{* \star}\right)\end{array}$ & $\begin{array}{c}1415379 \\
(1161893)\end{array}$ \\
\hline$P M G$ & $\begin{array}{l}643^{\star \star} \\
\left(653^{* \star}\right)\end{array}$ & $\begin{array}{l}4,2^{*} \\
\left(6^{\star \star}\right)\end{array}$ & $\begin{array}{l}128^{* *} \\
\left(68^{* *}\right)\end{array}$ & $\begin{array}{l}8^{* *} \\
\left(6^{* *}\right)\end{array}$ & $\begin{array}{l}1,4 \\
(2)\end{array}$ \\
\hline
\end{tabular}

Tableau IV. Corrélations entre les classements des parents obtenus dans les différents lieux; entre parenthèses, même chose pour les classements des hybrides obtenus dans les différents lieux.

\begin{tabular}{|c|c|c|c|c|}
\hline & & $R D T$ & NG & $P M G$ \\
\hline \multicolumn{2}{|c|}{$\begin{array}{l}\text { coefficient de concordance de } \\
\text { Kendall pour les } 3 \text { lieux }\end{array}$} & $\begin{array}{c}0,670 \\
(0,631)\end{array}$ & $\begin{array}{c}0,774 \\
(0,743)\end{array}$ & $\begin{array}{c}0,895 \\
(0,874)\end{array}$ \\
\hline \multirow{3}{*}{$\begin{array}{l}\text { coefficient } \\
\text { de corrélation } \\
\text { de rangs de } \\
\text { Spearman }\end{array}$} & Clermont-Moulon & $\begin{array}{c}0,612 \\
(0,606)\end{array}$ & $\begin{array}{c}0,723 \\
(0,676)\end{array}$ & $\begin{array}{c}0,875 \\
(0,849)\end{array}$ \\
\hline & Clermont - Mons & $\begin{array}{c}0,310 \\
(0,322)\end{array}$ & $\begin{array}{c}0,617 \\
(0,557)\end{array}$ & $\begin{array}{c}0,810 \\
(0,784)\end{array}$ \\
\hline & Moulon-Mons & $\begin{array}{c}0,591 \\
(0,413)\end{array}$ & $\begin{array}{c}0,670 \\
(0,610)\end{array}$ & $\begin{array}{c}0,826 \\
(0,800)\end{array}$ \\
\hline
\end{tabular}

Tableau V. Moyenne des écarts types interlieux pour les 7 caractères étudiés, d'une part pour les parents et les hybrides, d'autre part pour les valeurs d'hétérosis par rapport aux parents moyen et supérieur.

\begin{tabular}{lcccc}
\hline Parents & $\begin{array}{l}\text { Moyenne des écarts types interlieux pour } \\
\text { Hybrides }\end{array}$ & $\begin{array}{c}\text { Hétérosis par } \\
\text { rapport au } \\
\text { parent moyen }\end{array}$ & $\begin{array}{c}\text { Hétérosis par } \\
\text { rapport au } \\
\text { parent supérieur }\end{array}$ \\
\hline$R D T$ & 6,58 & 7,14 & 4,47 & 4,59 \\
$N E$ & 116 & 143 & 5,01 & 5,38 \\
$N G$ & 1388 & 1583 & 4,48 & 4,44 \\
$N G E$ & 6,37 & 8,7 & 5,93 & 5,79 \\
$P M G$ & 2,69 & 2,67 & 2,84 & 4,15 \\
$H T$ & 9,50 & 13,64 & 2,85 & 2,50 \\
$P R E C$ & 1,31 & 1,35 & 0,32 & 0,34 \\
\hline
\end{tabular}


Tableau VI. Analyses de variance sur les valeurs d'hétérosis par rapport au parent moyen des 3 lieux; entre parenthèses, même chose pour les valeurs d'hétérosis par rapport au parent supérieur. * = significatif au seuil de $5 \%$; ${ }^{* \star}=$ significatif au seuil de $1 \%$.

\begin{tabular}{|c|c|c|c|c|c|}
\hline & $\begin{array}{l}\text { lieu } \\
2 d d l \\
(2 d d l)\end{array}$ & $\begin{array}{l}\text { bloc } \\
6 d d l \\
(6 d d l)\end{array}$ & $\begin{array}{l}\text { Carrés moyens } \\
\text { génotype } \\
\\
34 d d l \\
\text { (34 ddl) }\end{array}$ & $\begin{array}{c}\text { interaction } \\
\text { lieu-génotype } \\
60 \mathrm{ddl} \\
(60 \mathrm{ddl})\end{array}$ & $\begin{array}{c}\text { erreur } \\
187 d d l \\
(187 d d l)\end{array}$ \\
\hline $\begin{array}{l}\text { hétérosis } \\
\text { RDT }\end{array}$ & $\begin{array}{c}31 \\
(42)\end{array}$ & $\begin{array}{c}112^{*} \\
\left(112^{*}\right)\end{array}$ & $\begin{array}{c}116^{* *} \\
\left(115^{* *}\right)\end{array}$ & $\begin{array}{c}74^{\star *} \\
\left(73^{* \star}\right)\end{array}$ & $\begin{array}{c}39 \\
(40)\end{array}$ \\
\hline $\begin{array}{l}\text { hétérosis } \\
\qquad N G\end{array}$ & $\begin{array}{c}92 \\
\left(170^{*}\right)\end{array}$ & $\begin{array}{c}206^{\star \star} \\
\left(167^{* *}\right)\end{array}$ & $\begin{array}{c}153^{\star *} \\
\left(229^{* *}\right)\end{array}$ & $\begin{array}{l}76^{* \star} \\
\left(71^{* *}\right)\end{array}$ & $\begin{array}{c}37 \\
(40)\end{array}$ \\
\hline $\begin{array}{l}\text { hétérosis } \\
P M G\end{array}$ & $\begin{array}{c}33 \\
\left(56^{*}\right)\end{array}$ & $\begin{array}{c}30 \\
\left(38^{*}\right)\end{array}$ & $\begin{array}{c}135^{\star *} \\
\left(338^{* *}\right)\end{array}$ & $\begin{array}{c}30^{* \star} \\
\left(63^{* \star}\right)\end{array}$ & $\begin{array}{c}15 \\
(16)\end{array}$ \\
\hline
\end{tabular}

Tableau VII. Cœefficients de corrélation linéaire mettant en évidence les liens existant entre le rendement de l'hybride ou l'hétérosis pour le rendement d'une part, et la stabilité interlieux de ce rendement ou de cet hétérosis d'autre part.

\begin{tabular}{|c|c|c|c|}
\hline & Clermont & Le Moulon & Mons \\
\hline $\begin{array}{l}\text { corrélation entre rendement de l'hybride et } \\
\text { écart type interlieux de ce rendement }\end{array}$ & $-0,359$ & $+0,592$ & $+0,063$ \\
\hline $\begin{array}{l}\text { corrélation entre rendement de l'hybride et } \\
\text { écart type interlieux de l'hétérosis PM }\end{array}$ & 0 & $+0,375$ & $-0,105$ \\
\hline $\begin{array}{l}\text { corrélation entre rendement de l'hybride et } \\
\text { écart type interlieux de l'hétérosis PS }\end{array}$ & $+0,313$ & $+0,418$ & $-0,032$ \\
\hline $\begin{array}{l}\text { corrélation entre hétérosis PM pour le } \\
\text { rendement et écart type de cet hétérosis }\end{array}$ & $-0,126$ & $+0,459$ & $-0,071$ \\
\hline $\begin{array}{l}\text { corrélation entre hétérosis PS pour le } \\
\text { rendement et écart type de cet hétérosis }\end{array}$ & 0 & $+0,167$ & $-0,158$ \\
\hline
\end{tabular}
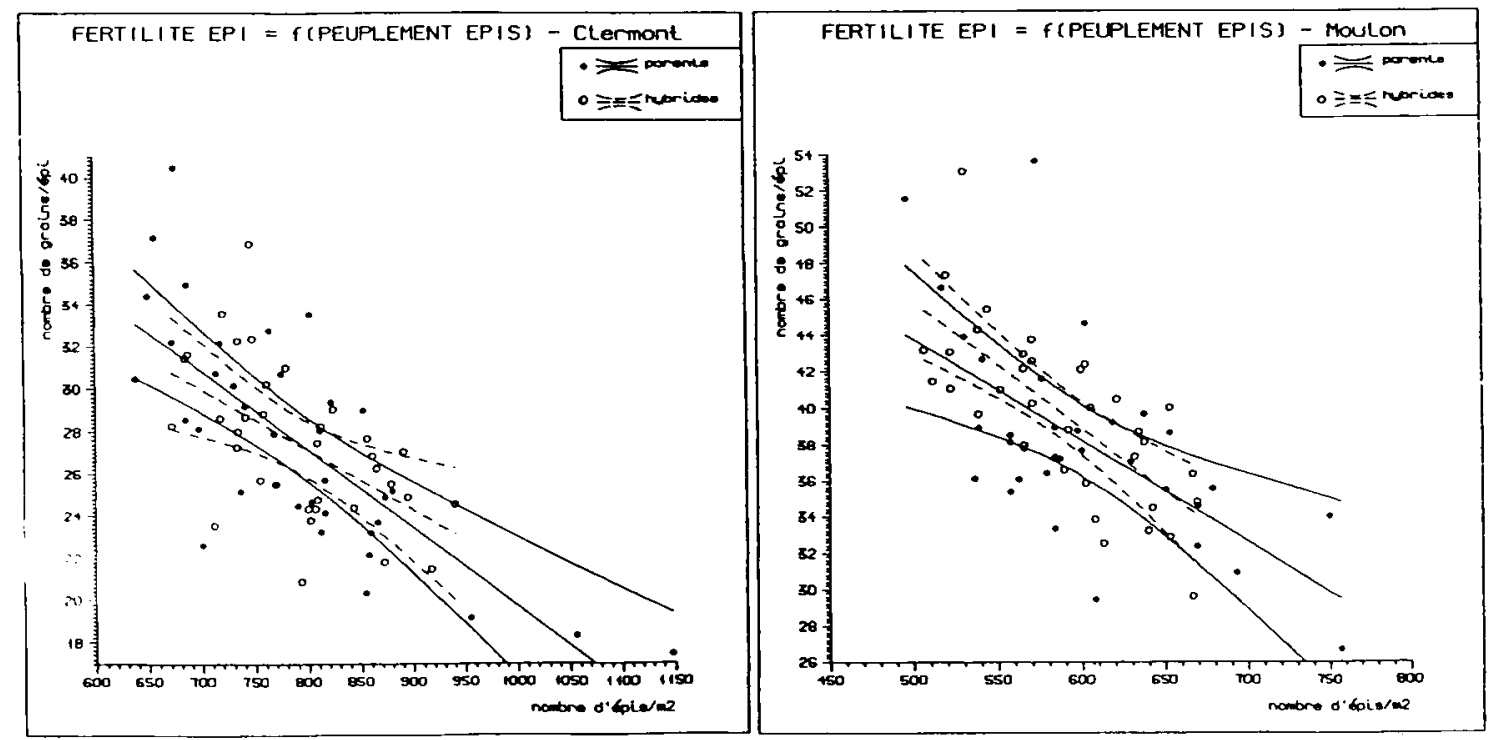

Fig 6. Régression linéaire du nombre de grains par épi sur le nombre d'épis par m2, pour les parents et les hybrides (les courbes encadrant les droites de régression délimitent la région de confiance à $95 \%$ de ces droites), en 2 lieux (Clermont et Le Moulon). 

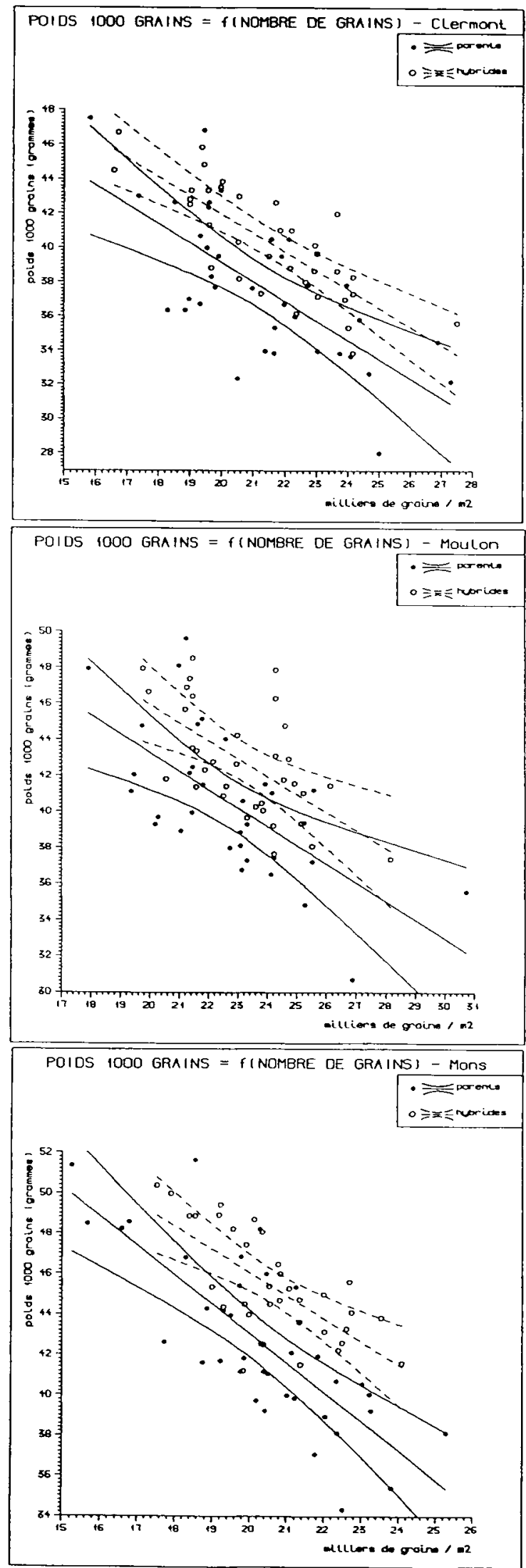

Fig 7. Régression linéaire du poids de 1000 grains sur le nombre de grains par $\mathrm{m}^{2}$, pour les parents et pour les hybides (les courbes encadrant les droites de régression délimitent la région de confiance à $95 \%$ de ces droites), dans les 3 lieux.

\section{Intérêt des blés hybrides}

Nos résultats indiquent qu'en conditions de culture intensive l'homéostasie des hybrides est équivalente à celle des lignées. Ceci avait déjà été souligné par Wienhues (1967), Carver et al (1987), et Smith et al (1987, 1988). En conséquence, l'évaluation multilocale et pluriannuelle des $F_{1}$ reste nécessaire. La stabilité théoriquement attendue chez l'hybride, du fait de la réunion de caractères de résistance aux diverses adversités (Auriau et al, 1979; Bingham, 1983; Lucken, 1986; Rousset, 1986), pourrait cependant apparaître dans des conditions de culture plus extensives. II serait donc intéressant de voir si les $F_{1}$ répondent différemment des lignées à une diminution des densités de semis, ou à des traitements phytosanitaires réduits.

En ce qui concerne le rendement, l'hétérosis par rapport au parent supérieur est au moins de $4 \%$ dans les 3 lieux, pour notre échantillon de 35 hybrides. II est du même ordre de grandeur que ceux rapportés dans la littérature : $4,3 \%$ pour 10 hybrides (Borghi et al, 1988), 7,3\% pour 17 hybrides (Brears et al, 1988), 3,6\% pour 430 hybrides (Morgan et al, 1989) et 3,1\% pour 34 hybrides (Oury et al, 1990). L'écart type des valeurs de l'hétérosis par rapport au parent supérieur pour le rendement est assez important : il vaut 5 à Clermont, 7,1 au Moulon et 6,1 à Mons. Il semble donc possible de faire une sélection des parents pour atteindre des niveaux d'hétérosis plus élevés. Toutefois, dans cette optique, le fait que, pour le rendement, l'hétérosis par rapport au parent supérieur diminue lorsque le rendement du parent supérieur augmente, n'est pas un élément favorable. Mais cette corrélation négative n'est significative que dans 2 lieux sur 3 , et n'est, de toute façon, pas très forte (tableau II).

Cependant, l'aspect le plus important par rapport à la possibilité de développement des blés hybrides n'est pas tant la manifestation nette d'une vigueur hybride au niveau du rendement, que l'existence d'une supériorité des meilleurs hybrides par rapport aux meilleures lignées. Dans notre expérimentation, cette supériorité est de l'ordre de $6 \%$, dans un contexte où on ne cherchait pas à mettre en évidence les potentialités maximales des $F_{1}$. Les travaux actuellement conduits sur les blés hybrides, qui portent maintenant sur des effectifs de $F_{1}$ suffisamment conséquents pour que les tendances dégagées aient une valeur statistique (Borghi et al, 1989; Morgan et al, 1989; Oury et al, 1990), pourraient 
Tableau VIII. Cœfficient de corrélation linéaire, d'une part entre le rendement et ses composantes, d'autre part entre la valeur de l'hétérosis pour le rendement et les valeurs de l'hétérosis pour les composantes de ce rendement; $a=$ Clermont; $\mathbf{b}=$ Le Moulon; $c=$ Mons

\section{Corrélations entre le rendement et ses composantes pour}

parents hybrides

Corrélations entre l'hétérosis pour le rendement et l'hétérosis pour les composantes du rendement

$\begin{array}{cl}\text { hétérosis par rapport } & \text { hétérosis par rapport } \\ \text { au parent moyen } & \text { au parent supérieur }\end{array}$

\begin{tabular}{|c|c|c|c|c|c|}
\hline$N E$ & $\begin{array}{l}a \\
b \\
c\end{array}$ & $\begin{array}{l}-0,290 \\
-0,130\end{array}$ & $\begin{array}{l}-0,144 \\
-0,236\end{array}$ & $\begin{array}{r}-0,166 \\
0,308\end{array}$ & $\begin{array}{l}0,051 \\
0,131\end{array}$ \\
\hline$N G$ & $\begin{array}{l}a \\
b \\
c\end{array}$ & $\begin{array}{l}0,479 \\
0,495 \\
0,507\end{array}$ & $\begin{array}{l}0,688 \\
0,540 \\
0,675\end{array}$ & $\begin{array}{l}0,572 \\
0,820 \\
0,644\end{array}$ & $\begin{array}{l}0,389 \\
0,754 \\
0,368\end{array}$ \\
\hline NGE & $\begin{array}{l}a \\
b \\
c\end{array}$ & $\begin{array}{l}0,514 \\
0,476\end{array}$ & $\begin{array}{l}0,674 \\
0,530\end{array}$ & $\begin{array}{l}0,431 \\
0,420\end{array}$ & $\begin{array}{l}0,328 \\
0,566\end{array}$ \\
\hline$P M G$ & $\begin{array}{l}a \\
b \\
c\end{array}$ & $\begin{array}{l}0,293 \\
0,336 \\
0,141\end{array}$ & $\begin{array}{r}-0,107 \\
0,340 \\
0,074\end{array}$ & $\begin{array}{r}0,502 \\
-0,093 \\
0,203\end{array}$ & $\begin{array}{r}0,101 \\
-0,112 \\
0,210\end{array}$ \\
\hline
\end{tabular}

permettre, par une meilleure connaissance du comportement des hybrides et le choix mieux raisonné des parents, d'accroitre l'écart entre les combinaisons hybrides les plus performantes et les meilleures lignées.

\section{RÉFÉRENCES}

Auriau P, Pluchard P, Chapuset T, Tallon P (1979) Quel est l'avenir des blés hybrides. Ann Amélior Plant 29, 131-144

Bachacou J, Masson JP, Millier C (1981) Manuel de la Programmathèque Statistique AMANCE. INRA, $516 \mathrm{p}$

Bingham $J(1983)$ Genetic constraints on progress in wheat breeding. In: The yields of cereals, Proc Int Seminar, Camb and NAC Stamleigh 5-11

Borghi B, Perenzin M, Nash RJ (1988) Agronomic and qualitative characteristics of ten bread wheat hybrids produced using a chemical hybridizing agent. Euphytica 39, 185-194

Borghi B, Perenzin M, Nash RJ (1989) Combining ability estimates in bread wheat and performance of 100 hybrids produced using a chemical hybridizing agent. J Genet \& Breed 43, 11-16

Brears T, Hydon AG, Bingham J (1988) An assessment of the feasibility of producing $F_{1}$ and $F_{2}$ hybrids for the UK. In: Proc 7th int Wheat Genet Symp, 1057-1062

Carver BF, Smith EL, England HO Jr (1987) Regression and Cluster Analysis of Environnemental Res- ponses of Hybrid and Pureline Winter Wheat Cultivars. Crop Sci 27, 659-664

Glenn DE (1987) Seed wheat production: varieties and hybrids. In: Hybrid and conventionnal variety selection considerations, proc 2nd Nat Wheat Res Conf, Kansas City, february 1987, $10 p$

Goujon C, Paquet J (1968) Contribution à l'étude de la vigueur hybride chez le blé tendre : essais de rendements en semis d'automne réalisés en 196566 et 1966-1967. Ann Amélior Plant 18, 223-235

Keydel F (1973) Untersuchung über die Leistungseigenschaften von $F_{1}$-Hybriden von Winterweizen. $Z$ Pflanzenzuecht 69, 239-255

Lucken KA (1986) The breedling and production of hybrid wheat. In: Genetic improvement in yield of wheat, proc CSSA Symp special publ, 87-107

Morgan CL, Austin RB, Ford MA, Bingham J, Angus WJ, Chowdhury $S$ (1989) An evaluation of $F_{1}$ hybrid winter wheat genotypes produced using a chemical hybridizing agent. J Agric Sci Cam 112, 143149

Oury FX, Koenig J, Bérard P, Rousset M (1990) Une comparaison entre des blés hybrides produits par voie chimique et leurs parents : niveaux d'hétérosis et élaboration du rendement. Agronomie 10, 291. 304

Rousset M (1986) Les blés hybrides sortent du laboratoire. Recherche 17, 86-89

Smith EL, Sharma RC, Morgan GH, Carver BF, Merrit RG (1987) Results of 1986 wheat variety-hybrid performance test. Research report p-889, Okla Agric Exp Stn, Rep 22 p 
Tomassone R, Lesquoy E, Millier C (1983) La régression : nouveaux regards sur une ancienne méthode statistique. INRA et Masson, Paris, $180 \mathrm{p}$

Triboi E (1986) Étude du potentiel génétique de production du blé tendre à travers la structure et le fonctionnement du peuplement. In: Rapport intermédiaire de I'ATP Ecophysiologie du Blé, INRA, 93-103
Triboi E, Blanchon J, Magne J (1985) Déterminisme du poids moyen du grain chez le blé. Effet sur la variation du rendement. $C R$ Seances Acad Agric Fr $71,871-886$

Wienhues $F$ (1967) Long-term yield analyses about heterosis in wheat and barley: variability of heterosis, fixation of heterosis. Communication au congrès Eucarpia, Wageningen, octobre 1967. 